\title{
Tooth Discoloration and Internal Bleaching after the use of Triple Antibiotic Paste for Regenerative Endodontic Treatment of an Immature Necrotic Permanent Tooth: A Case Report
}

\section{Gayathri Parthiban*, Rupali Karale, Vinay Kumar and Prashanth BR}

Department of Conservative Dentistry and Endodontics, KLE Society's Institute of Dental Sciences, Bangalore, Karnataka, India

*Corresponding author: Gayathri Parthiban, Department of Conservative Dentistry and Endodontics, KLE Society's Institute of Dental Sciences, Bangalore, 20, Tumkur Road, 2nd Stage, Yeshwanthpur Suburb, Bengaluru, Karnataka, 560022, India, Tel: +91 8884560235; E-mail: gayathri.pvm@gmail.com

Received date: Dec 24, 2018; Accepted date: Jan 01, 2019; Published date: Jan 05, 2019

Copyright: @2018 Parthiban G, et al. This is an open-access article distributed under the terms of the Creative Commons Attribution License, which permits unrestricted use, distribution, and reproduction in any medium, provided the original author and source are credited.

\begin{abstract}
Recently, regeneration of immature necrotic pulp has become an alternative conservative treatment option for young permanent teeth and is a subject of great interest in the field of endodontics. The primary goal of these procedures is to activate the potential of the pulp for dentine deposition and produce a stronger mature root that withstands the forces better. However, the current protocol has potential clinical and biological complications. Amongst them, crown discoloration caused by triple antibiotic paste is deemed to be very frustrating for the dentist and the patient. In the case presented, there was a successful reversal of the crown discoloration by an internal bleaching procedure with superoxol at follow up periods of 3,6 and 12 months. However, further long-term clinical trials and histological studies are needed to evaluate the long-term outcomes of walking bleach with superoxol in regenerative endodontic procedures.
\end{abstract}

Keywords: Discoloration; Immature teeth; Minocycline; Regenerative endodontics; Revascularization; Triple antibiotic paste

\section{Introduction}

Endodontic treatment of an immature necrotic tooth with arrested root development is significantly more difficult as the thin root walls are susceptible to fracture and the disinfection in these cases often relies on irrigation and intracanal medicaments $[1,2]$. In the present case, the regenerative procedure was performed on an immature necrotic permanent tooth using Platelet Rich Fibrin (PRF) [3]. Walking bleach with superoxol was carried out as an economical, predictable and rather quick treatment option to reverse the discoloration caused by triple antibiotic paste.

\section{Case History}

A 14-year-old boy reported with a fracture with respect to tooth 11 . There was a history of trauma 6 months back due to fall. The medical status was non-contributory. On intraoral examination, the tooth 11 had Ellis and Davey's class IV fracture. Clinically, the crown exhibited very mild discoloration (Figure 1).

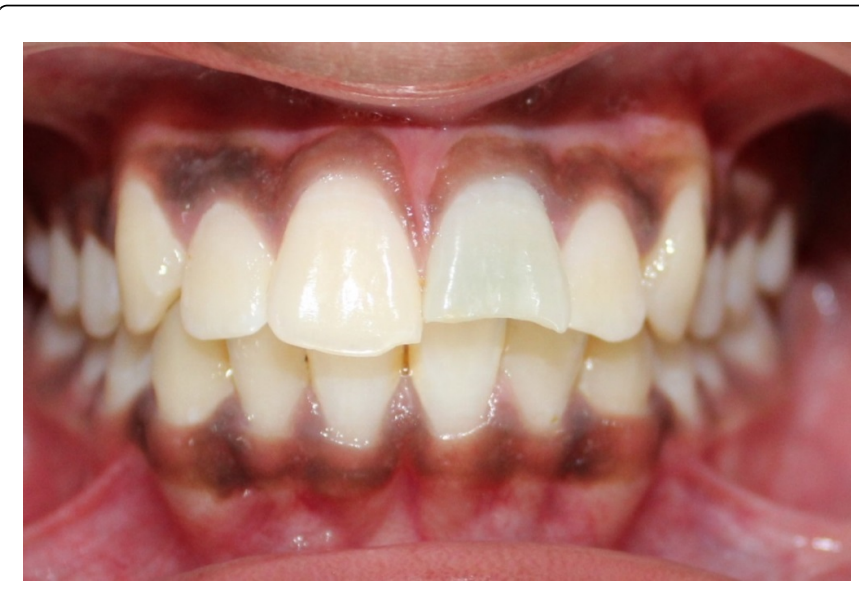

Figure 1: Pre-operative clinical picture of the crown exhibiting very mild discoloration.

The tooth was tender on percussion and did not respond to cold and electric pulp test. Periodontal probing depth was within normal limits. On radiographic examination, the tooth showed an incompletely formed root, thin dentinal walls with the open apex (Cvek's stage 4 root development) and associated periapical radiolucency (Figure 2). 
Citation: Parthiban G, Karale R, Kumar V, Prashanth BR (2018) Tooth Discoloration and Internal Bleaching after the use of Triple Antibiotic Paste for Regenerative Endodontic Treatment of an Immature Necrotic Permanent Tooth: A Case Report. Dentistry 8: 528 . doi: $10.4172 / 2161-1122.1000528$

Page 2 of 4

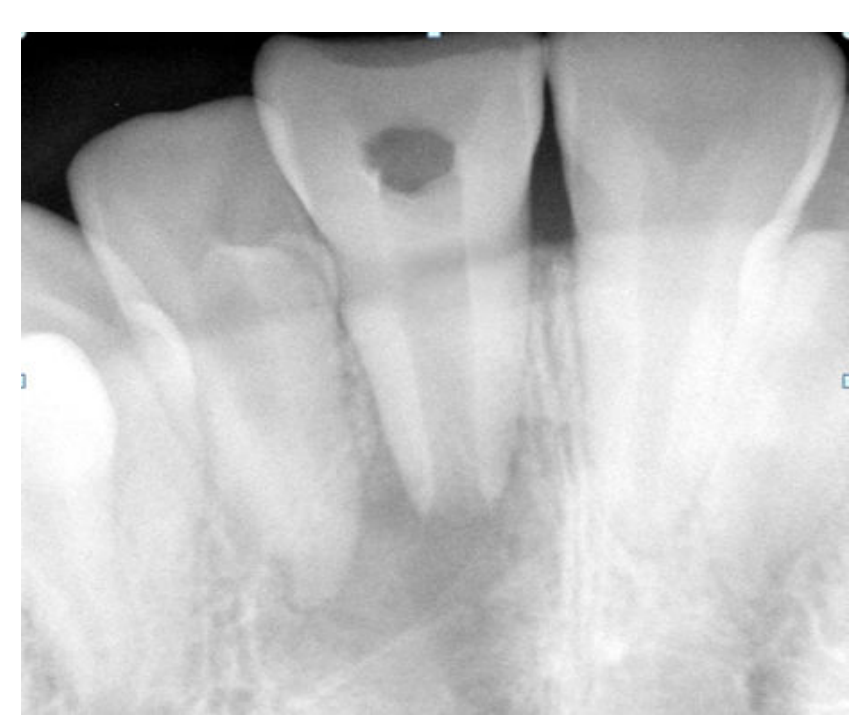

Figure 2: Radiograph showing open apex and periapical radiolucency.

It was diagnosed as pulp necrosis with asymptomatic apical periodontitis and was planned for a regenerative endodontic treatment using PRF as a scaffold. The treatment protocol was narrated to the parents and written informed consent was taken.

Local anesthesia was given using 2\% Lidocaine with 1:80000 epinephrine and rubber dam application was done. After access opening, the necrotic pulp was removed, and the pus was allowed to drain completely. The canal was thoroughly irrigated using $20 \mathrm{ml}$ of $1.5 \% \mathrm{NaOCl}$ for $5 \mathrm{~min}$ and then irrigated with $20 \mathrm{ml}$ saline for $5 \mathrm{~min}$, with a 27-gauge needle positioned about $1 \mathrm{~mm}$ from the root end. Working length was determined radiographically and was confirmed with the help of an apex locator. The canal was dried using paper points. A thick mixture of Triple antibiotic paste was placed inside the canal to a depth of two $\mathrm{mm}$ short of the apex and one $\mathrm{mm}$ below the $\mathrm{CEJ}$ using an endodontic plugger.

The patient was recalled after 28 days. The patient was asymptomatic but mild discoloration of the crown was noted. The canal was copiously irrigated using saline and $1.5 \% \mathrm{NaOCl}$ to remove the antibiotic paste. Then $20 \mathrm{~mL}$ of $17 \%$ EDTA was used and the root canal was dried with paper points.

For the preparation of PRF, $9 \mathrm{ml}$ of blood was drawn and centrifuged (Remi Model, Mumbai, India) The PRF membrane was cut into small fragments using scalpel blade and placed incrementally inside the canal using an endodontic hand plugger (Dentsply Maillefer, Switzerland). Then 3 to $4 \mathrm{~mm}$ of MTA (MTA, Angelus, Brazil) was placed directly over the PRF membrane and a wet cotton pellet was placed, and the access cavity was sealed with GIC (Figure 3).

At the 3-month recall, the patient was asymptomatic. However, Blue-grayish discoloration of coronal tooth structure was noted (Figure 4).

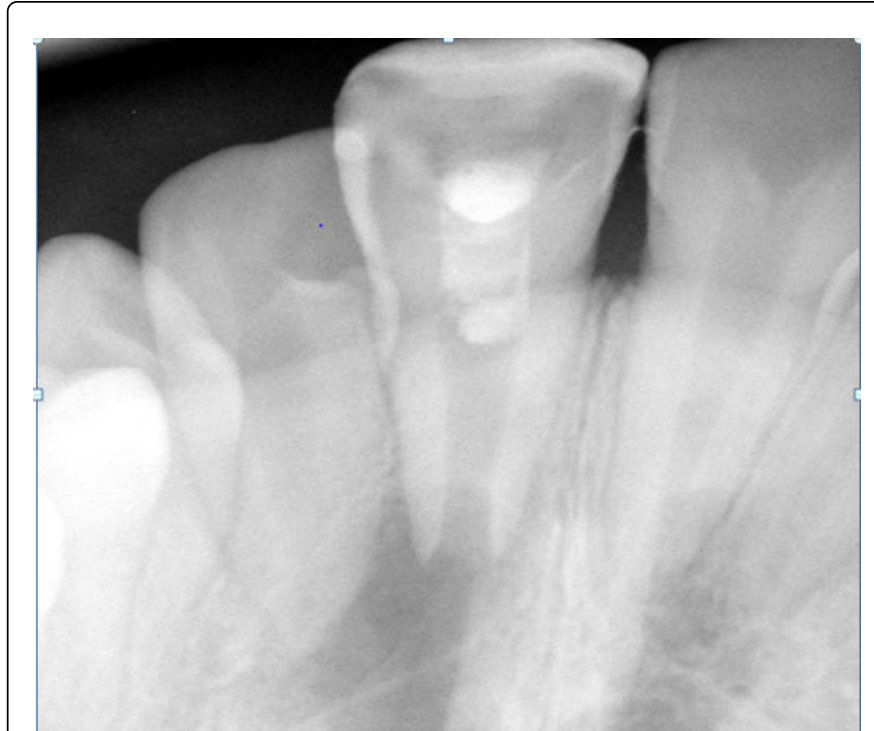

Figure 3: Radiograph taken immediately after placement of PRF.

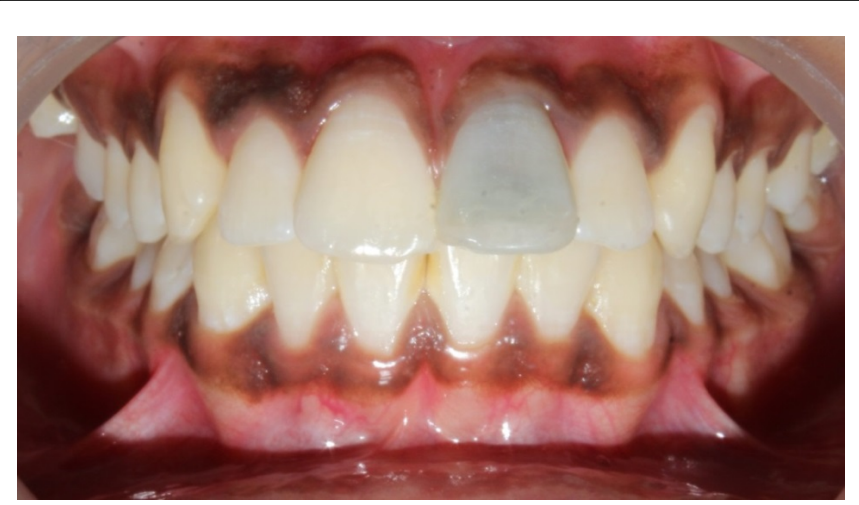

Figure 4: Clinical picture of the discolored crown.

Walking bleach procedure was planned to resolve the esthetic problem. A baseline shade was taken with a shade guide (VITA Toothguide 3D-Master). After placing an intra orifice barrier $2 \mathrm{~mm}$ below the cementoenamel junction with a resin-modified glass ionomer cement, a bleaching agent (Sodium perborate mixed with $30 \%$ hydrogen peroxide in a ratio of $2 \mathrm{~g}: 1 \mathrm{~mL}$ ) in a thick consistency was placed in the pulp chamber and sealed with a temporary filling (MD-Temp). Significant improvement in the shade was noticed soon after placement of the bleaching agent.

Calcium hydroxide powder was mixed with saline and placed in the access cavity and sealed with a temporary filling (MD-Temp). After a week, the access cavity was filled with a bonded composite resin restoration. Follow-ups were done at 6-and 12-months period. The patient was asymptomatic, the bleached shade was maintained (Figure 5) and radiographs showed resolution of periapical radiolucency (Figure 6). The case is under observation for the further outcome of the regenerative procedure. 
Citation: Parthiban G, Karale R, Kumar V, Prashanth BR (2018) Tooth Discoloration and Internal Bleaching after the use of Triple Antibiotic Paste for Regenerative Endodontic Treatment of an Immature Necrotic Permanent Tooth: A Case Report. Dentistry 8: 528. doi: $10.4172 / 2161-1122.1000528$

Page 3 of 4

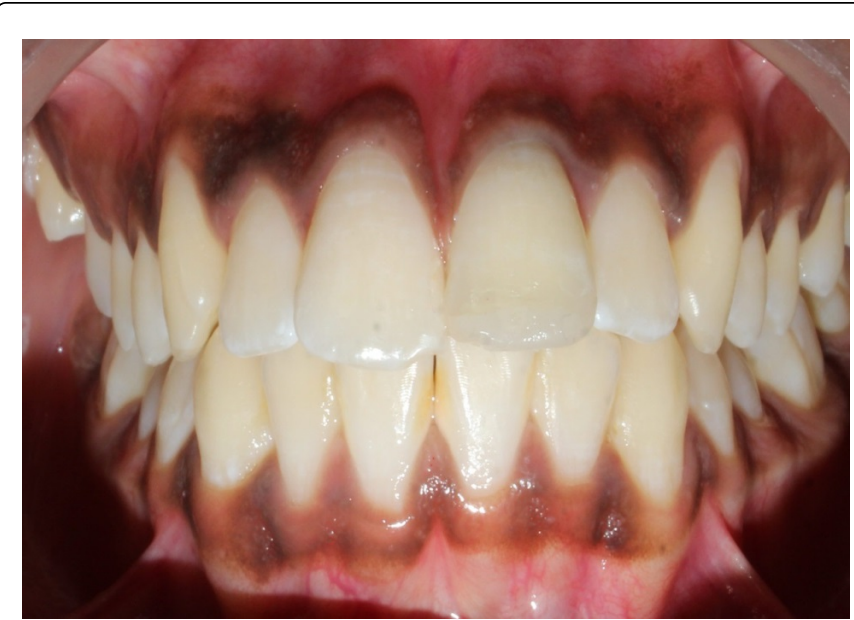

Figure 5: Immediate post-operative shade retained at the end of 12 months.

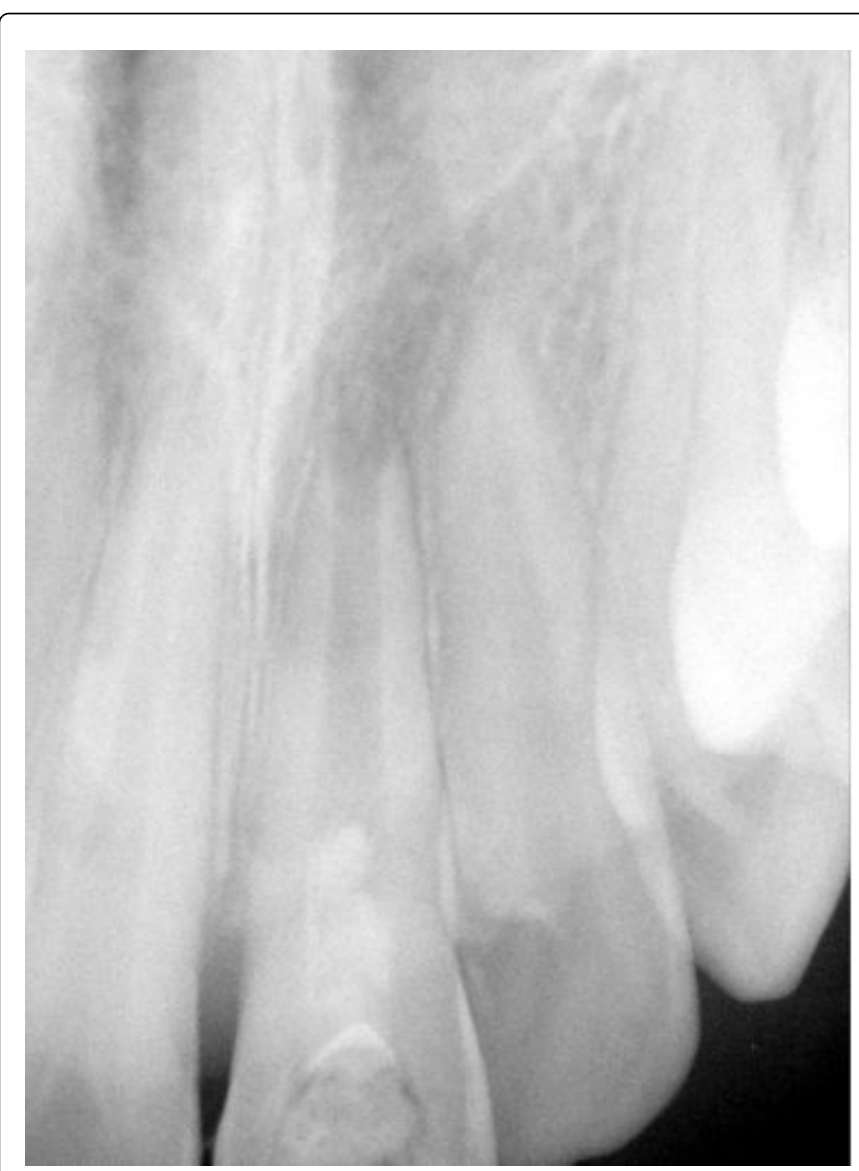

Figure 6: Radiograph showing resolution of periapical radiolucency at the end of 12 months.

\section{Discussion}

Regenerative procedure in immature teeth was introduced in the field of endodontics by Ostby in 1961 and later reintroduced in 1966 by
Rule and Winter [4]. The second-generation platelet concentrate PRF was used in this case to overcome the limitations associated with both conventional blood clot and other matrices like PRP, collagen etc. [5] MTA was placed over the PRF scaffold, as it sets in the presence of blood and does not lead to leakage [6].

The triple-antibiotic paste is proved to be an effective disinfectant and is the most commonly used medicament $[7,8]$. However, the major disadvantage associated with its use is the crown discoloration resulting from minocycline [5]. Therefore, in the present case care was taken to keep the paste one mm below the CEJ of the tooth. Despite the precautionary measure, there was blue-grayish discoloration of the crown at the end of three months. Application of bonding agent to the pulp chamber walls before placement of the triple antibiotic paste is a suggested method to reduce the discoloration. However, it has been shown that it can only reduce the change in darkness of the teeth but cannot prevent it [9].

With the conventional walking bleach technique (Sodium perborate and saline) the results may vary depending on the type of discoloration and time period the tooth has been discolored. It may take up to four appointments and a month for significant improvement in the shade [8-10]. However in the case presented, satisfactory results were obtained immediately on the same appointment. This could be attributed to the $30 \%$ hydrogen peroxide (Superoxol) which was used in combination with sodium perborate. A $2 \mathrm{~mm}$ intraorifice barrier using RMGIC was placed to prevent cervical resorption. In addition, calcium hydroxide powder was placed in the chamber as a medicament for a period of one week before placing the permanent restoration to prevent cervical resorption. At the end of one year, there was no significant alteration in the bleached shade and radiograph showed resolution of periapical radiolucency.

\section{Conclusion}

The present case report highlights the resolution of crown discoloration caused due to triple antibiotic paste used in a regenerative endodontic treatment using Platelet-Rich Fibrin (PRF) as a scaffold, in an immature tooth with necrotic pulp and periapical pathosis.

\section{References}

1. Sachdeva GS, Sachdeva LT, Goel M, Bala S (2015) Regenerative endodontic treatment of an immature tooth with a necrotic pulp and apical periodontitis using platelet-rich plasma (PRP) and mineral trioxide aggregate (MTA): A case report. Int Endod J 48: 902-910.

2. Galler KM (2016) Clinical procedures for revitalization: Current knowledge and considerations. Int Endod J 49: 926-936.

3. Dohan DM, Choukroun J, Diss A, Dohan SL, Dohan AJJ, et al. (2006) Platelet-rich fibrin (PRF): a second-generation platelet concentrate. Part I: technological concepts and evolution. Oral Surg Oral Med Oral Pathol Oral Radiol Endod 101: e37-e44.

4. Mishra N, Narang I, Mittal N (2013) Platelet-rich fibrin-mediated revitalization of immature necrotic tooth. Contemp Clin Dent 4: 412-415.

5. Nagaveni NB, Pathak S, Poornima P, Joshi JS (2016) Revascularization Induced Maturogenesis of non-vital immature permanent tooth using platelet-rich-fibrin: A case report. J Clin Pediatr Dent 40: 26-30.

6. Belobrov I, Parashos P (2011) Treatment of tooth discoloration after the use of white mineral trioxide aggregate. J Endod 37: 1017-1020.

7. Windley W, Teixeira F, Levin L, Sigurdsson A, Trope M (2005) Disinfection of immature teeth with a triple antibiotic paste. J Endod 31: 439-443. 
Citation: Parthiban G, Karale R, Kumar V, Prashanth BR (2018) Tooth Discoloration and Internal Bleaching after the use of Triple Antibiotic Paste for Regenerative Endodontic Treatment of an Immature Necrotic Permanent Tooth: A Case Report. Dentistry 8: 528. doi: $10.4172 / 2161-1122.1000528$

Page 4 of 4

8. Kirchhoff AL, Raldi DP, Salles AC, Cunha RS, Mello I (2015) Tooth discolouration and internal bleaching after the use of triple antibiotic paste. Int Endod J 48: 1181-1187.

9. Kim JH, Kim Y, Shin SJ, Park JW, Jung IY (2010) Tooth discoloration of immature permanent incisor associated with triple antibiotic therapy: a case report. J Endod 36: 1086-1091.
10. Kahler B, Rossi Fedele G (2016) A Review of tooth discoloration after regenerative endodontic therapy. J Endod 42: 563-569. 